\title{
JORDAN DERIVATIONS OF PRIME RINGS ${ }^{1}$
}

\author{
I. N. HERSTEIN
}

1. Given any associative ring $A$ one can construct from its operations and elements a new ring, the Jordan ring of $A$, by defining the product in this ring to be $a \circ b=a b+b a$ for all $a, b \in A$, where the product $a b$ signifies the product of $a$ and $b$ in the associative ring $A$ itself.

If $R$ is any ring, associative or otherwise, by a derivation of $R$ we shall mean a function, ', mapping $R$ into itself so that

$$
\begin{aligned}
(r+s)^{\prime} & =r^{\prime}+s^{\prime}, \\
(r s)^{\prime} & =r^{\prime} s+r s^{\prime}
\end{aligned}
$$

for all $r, s \in R$.

By a Jordan derivation, ', of an associative ring $A$ we shall mean a derivation of the Jordan ring formed from $A$; that is, for all $a, b \in A$, $(a \circ b)^{\prime}=a^{\prime} \circ b+a \circ b^{\prime}$. Writing this out in terms of the associative operations of $A$, a Jordan derivation, ', of $A$ is an additive mapping which satisfies $(a b+b a)^{\prime}=a^{\prime} b+a b^{\prime}+b^{\prime} a+b a^{\prime}$ for all $a, b \in A$.

In this paper we show that for a rather wide class of rings, namely prime rings of characteristic different from 2 (i.e. $2 x=0$ implies $x=0$ ) a Jordan derivation of $A$ is automatically an ordinary derivation of $A$. The converse is, of course, trivially true. In the last part of the paper we modify the definition of a Jordan derivation in such a way that the definition given above is still the definition if the ring has characteristic different from 2, but which, in characteristic 2, (except for a very special case) enables us to obtain the result that a Jordan derivation, irrespective of the characteristic, is an ordinary derivation whenever the ring is a prime ring.

For most of this paper all rings considered will be of characteristic not 2 ; for such rings $A$ a Jordan derivation, ', can be defined by

$$
\begin{aligned}
(a+b)^{\prime} & =a^{\prime}+b^{\prime}, \\
\left(a^{2}\right)^{\prime} & =a a^{\prime}+a^{\prime} a
\end{aligned}
$$

for all $a, b \in A$.

If a mapping * satisfied $(a+b)^{*}=a^{*}+b^{*}$ and $(a b)^{*}=b^{*} a+b a^{*}$, it would certainly be a Jordan derivation but it would, in general, not

Received by the editors April 8, 1957.

1 The research for this paper was supported, in part, by an ONR grant to Yale University and in part by an NSF grant to the author. The idea of a Jordan derivation was first raised in a conversation by Professor Irving Kaplansky. 
be a derivation. Let us call such a mapping a reverse derivation. Since our aim is to show that Jordan derivations are, for prime rings, coincident with ordinary derivations, one of our first aims will be to show that reverse derivations do not, in general, exist in prime rings.

We begin with a lemma which holds in any arbitrary associative ring, namely,

Lemma 1.1. Let $A$ be any ring; for $a \in A$ let $T(a)=\{r \in A \mid r(a x-x a)$ $=0$ for all $x \in A\}$. Then $T(a)$ is a two-sided ideal of $A$.

Proof. Clearly $T(a)$ is a left ideal of $A$. There remains but to show that if $u \in T(a), x \in A$, then $u x \in T(a)$. But then, for all $r \in A$, $u(a x r-x r a)=0$. Thus $u\{(a x-x a) r+x(a r-r a)\}=0$. Since $u \in T(a)$, $u(a x-x a)=0$, and so we have that $u x(a r-r a)=0$ for all $r \in A$. But then $u x \in T(a)$, whereby the lemma is proved.

We recall a ring $A$ is said to be a prime ring if $x A y=(0)$ for $x, y \in A$ implies that either $x=0$ or $y=0$. Equivalently, a prime ring is one where the right annihilator of a nonzero right ideal is merely (0).

We now have

Lemma 1.2. If $A$ is a prime ring and if $a \in A$ is not in $Z$, the center of $A$, then $T(a)=(0)$.

Proof. Since $a \notin Z$, for some $b \in A, a b-b a \neq 0$. If $T(a) \neq(0)$, then since $T(a)(a b-b a)=(0)$, the right ideal $T(a)$ would be annihilated by a nonzero element, which, by the definition of a prime ring, excludes the possibility that $T(a) \neq(0)$. So $T(a)=(0)$.

2. Reverse derivations of prime rings. In this section we dispose of the possibility of the occurrence of reverse derivations in prime rings with

Theorem 2.1. Let $A$ be a prime ring and suppose that $*$ is a nonzero reverse derivation of $A$. Then $A$ is a commutative integral domain and * is an ordinary derivation of $A$.

Proof. Let $a, b, c \in A$. Thus

$$
(a(b c))^{*}=(b c)^{*} a+(b c) a^{*}=c^{*} b a+c b^{*} a+b c a^{*} .
$$

Also

$$
((a b) c)^{*}=c^{*}(a b)+c(a b)^{*}=c^{*}(a b)+c b^{*} a+c b a^{*} .
$$

However, $a(b c)=(a b) c$, so the results of (1) and (2) must be equal. Thus, on comparing them, we obtain

$$
c^{*}(a b-b a)=(b c-c b) a^{*} \quad \text { for all } a, b, c \in A .
$$


If, in (3), we put $b=c$ we see that

$$
b^{*}(a b-b a)=0 \quad \text { for all } a \in A .
$$

But then $b^{*} \in T(b)$. Hence if $b^{*} \neq 0, T(b)$ must be different from (0). By Lemma 1.2 this yields that $b \in Z$, the center of $A$. That is, if $b \in A$ is such that $b^{*} \neq 0$ then $b \in Z$.

Suppose now that $b \in A$ is such that $b^{*}=0$. Since $*$ is not the zero mapping, there is an $a \in A$ so that $a^{*} \neq 0$. By the paragraph above, $a \in Z$. Also $(a+b)^{*}=a^{*}+b^{*}=a^{*} \neq 0$, so, again, $a+b \in Z$. Consequently it follows that $b \in Z$. Thus if $b^{*}=0$ or if $b^{*} \neq 0$ the net result is that $b \in Z$. Thus $A=Z$, so $A$ must be commutative; since it is a prime ring, it must then be a commutative integral domain. But then $(a b)^{*}=b^{*} a+b a^{*}=a b^{*}+a^{*} b$, so it is an ordinary derivation of $A$.

3. Jordan derivations of prime rings. The aim of this section is to establish that any Jordan derivation of a prime ring of characteristic $\neq 2$ is a derivation. We assume throughout this section that $A$ is a prime ring of characteristic $\neq 2$.

Lemma 3.1. If ' is a Jordan derivation of $A$ then for all $a, b \in A$, $(a b a)^{\prime}=a^{\prime} b a+a b^{\prime} a+a b a^{\prime}$.

Proof. Since $\left(a^{2}\right)^{\prime}=a a^{\prime}+a^{\prime} a$ when we linearize this by replacing $a$ by $a+b$ we obtain

$$
(a b+b a)^{\prime}=a^{\prime} b+a b^{\prime}+b^{\prime} a+b a^{\prime} \quad \text { for all } a, b \in A .
$$

Consider $W=(a(a b+b a)+(a b+b a) a)^{\prime}$. On the one hand, using (1) we see that

$$
\begin{aligned}
W= & a^{\prime}(a b+b a)+a(a b+b a)^{\prime}+(a b+b a)^{\prime} a+(a b+b a) a^{\prime} \\
= & a^{\prime} a b+a^{\prime} b a+a\left(a^{\prime} b+a b^{\prime}+b^{\prime} a+b a^{\prime}\right)+\left(a^{\prime} b+a b^{\prime}+b^{\prime} a+b a^{\prime}\right) a \\
& +(a b+b a) a^{\prime} \\
= & a^{\prime} a b+2 a^{\prime} b a+a a^{\prime} b+a^{2} b^{\prime}+2 a b a^{\prime}+2 a b^{\prime} a+b a a^{\prime}+b^{\prime} a^{2}+b a^{\prime} a .
\end{aligned}
$$

On the other hand,

$$
\begin{aligned}
W & =\left\{\left(a^{2} b+b a^{2}\right)+2 a b a\right\}^{\prime}=\left(a^{2}\right)^{\prime} b+a^{2} b^{\prime}+b^{\prime} a^{2}+b\left(a^{2}\right)^{\prime}+2(a b a)^{\prime} \\
& =a^{\prime} a b+a a^{\prime} b+a^{2} b^{\prime}+b^{\prime} a^{2}+b a a^{\prime}+b a^{\prime} a+2(a b a)^{\prime} .
\end{aligned}
$$

Comparing the two expressions, so obtained, for $W$ we arrive at $2(a b a)^{\prime}=2\left(a^{\prime} b a+a b^{\prime} a+a b a^{\prime}\right)$, and since the characteristic of $A$ is not $2,(a b a)^{\prime}=a^{\prime} b a+a b^{\prime} a+a b a^{\prime}$, which is the contention of the lemma.

We linearize the result of Lemma 3.1 by replacing $a$ by $a+c$ and so arrive at 
Lemma 3.2. For all $a, b, c \in A,(a b c+c b a)^{\prime}=a^{\prime} b c+c^{\prime} b a+a b^{\prime} c+c b^{\prime} a$ $+a b c^{\prime}+c b a^{\prime}$.

We now turn to the key

Lemma 3.3. For all $a, b \in A,\left((a b)^{\prime}-a^{\prime} b-a b^{\prime}\right)(a b-b a)=0$.

Proof. Consider $W=(a b(a b)+(a b) b a)^{\prime}$. Using Lemma 3.2, with $c=a b$ we obtain

$$
W=(a b)(a b)^{\prime}+(a b) b a^{\prime}+a b^{\prime}(a b)+(a b) b^{\prime} a+a^{\prime} b(a b)+(a b)^{\prime} b a .
$$

However,

$$
\begin{aligned}
W & =\left((a b)^{2}+a b^{2} a\right)^{\prime}=\left((a b)^{2}\right)^{\prime}+\left(a b^{2} a\right)^{\prime} \\
& =(a b)(a b)^{\prime}+(a b)^{\prime}(a b)+a^{\prime} b^{2} a+a\left(b^{2}\right)^{\prime} a+a b^{2} a^{\prime} \\
& =(a b)(a b)^{\prime}+(a b)^{\prime} a b+a^{\prime} b^{2} a+a b b^{\prime} a+a b^{\prime} b a+a b^{2} a^{\prime}
\end{aligned}
$$

by Lemma 3.1 .

Comparing the two expressions so obtained for $W$, we see that

$$
a b^{\prime}(a b)+a^{\prime} b(a b)+(a b)^{\prime}(b a)=(a b)^{\prime}(a b)+a^{\prime} b^{2} a+a b^{\prime}(b a) .
$$

Transposing and collecting terms, it follows that

$$
\left((a b)^{\prime}-a^{\prime} b-a b^{\prime}\right)(a b-b a)=0 .
$$

On linearizing the result of Lemma 3.3 we reach

Lemma 3.4. For all $a, b, c \in A$

$$
\left\{(a b)^{\prime}-a^{\prime} b-a b^{\prime}\right\}(c b-b c)+\left\{(c b)^{\prime}-c^{\prime} b-c b^{\prime}\right\}(a b-b a)=0
$$

and

(2) $\left\{(a b)^{\prime}-a^{\prime} b-a b^{\prime}\right\}(c a-a c)-\left\{(c a)^{\prime}-c^{\prime} a-c a^{\prime}\right\}(b a-a b)=0$.

Suppose that $a b=b a$. The first identity in the statement of Lemma 3.4 then reduces to $\left((a b)^{\prime}-a^{\prime} b-a b^{\prime}\right)(c b-b c)=0$ for all $c \in A$. Thus $(a b)^{\prime}-a^{\prime} b-a b^{\prime} \in T(b)$. If $b \notin Z$, by Lemma $1.2, T(b)=(0)$ and so it would follow that $(a b)^{\prime}-a^{\prime} b-a b^{\prime}=0$. Similarly, using the second identity in the statement of Lemma 3.4, if $a \in Z$ it would follow that $(a b)^{\prime}-a^{\prime} b-a b^{\prime}=0$. So suppose that both $a, b \in Z$. If $A=Z$, that is, if $A$ is commutative, then since $(a b+b a)^{\prime}=a^{\prime} b+a b^{\prime}+b^{\prime} a+b a^{\prime}$, $2(a b)^{\prime}=2\left(a^{\prime} b+a b^{\prime}\right)$ for all $a, b \in A$ and so $(a b)^{\prime}=a^{\prime} b+a b^{\prime}$. So we suppose that $A \neq Z$. Then there is a $c \in A, c \neq Z$. From the above, since $c \notin Z, a+c \notin Z$, and since $b c=c b, b(a+c)=(a+c) b,(c b)^{\prime}=c^{\prime} b+c b^{\prime}$, $(c b+a b)^{\prime}=((c+a) b)^{\prime}=(c+a)^{\prime} b+(c+a) b^{\prime}=c^{\prime} b+c b^{\prime}+a^{\prime} b+a b^{\prime}$. Thus we conclude here also that $(a b)^{\prime}=a^{\prime} b+a b^{\prime}$. We have proved 
Lemma 3.5. If $a, b \in A$ and if $a b=b a$ then $(a b)^{\prime}=a^{\prime} b+a b^{\prime}$.

Lemma 3.6. If $a, b \in A$ and if $a b=0$ then $0=(a b)^{\prime}=a^{\prime} b+a b^{\prime}$.

Proof. If $b a=0$ then $a b=b a=0$, which, by Lemma 3.5, implies that $0=(a b)^{\prime}=a^{\prime} b+a b^{\prime}$.

So we suppose that $b a \neq 0$. By Lemma 3.4

$$
\left((a b)^{\prime}-a^{\prime} b-a b^{\prime}\right)(c a-a c)+\left((c a)^{\prime}-c^{\prime} a-c a^{\prime}\right)(b a-a b)=0 .
$$

If we multiply (1) from the right by $b$, since $a b=0$, it reduces to

$$
\left((a b)^{\prime}-a^{\prime} b-a b^{\prime}\right) a c b=0 \quad \text { for all } c \in A .
$$

Since $b a \neq 0, b \neq 0$. Since we are in a prime ring (2) implies that $\left((a b)^{\prime}-a^{\prime} b-a b^{\prime}\right) a=0$. That is, since $a b=0$,

$$
\left(a^{\prime} b+a b^{\prime}\right) a=0 .
$$

Now (3) holds for all $b$ so that $a b=0$. If $a b=0$ then for all $r \in A$, $a(b r b)=0$. Using $b r b$ for $b$ in (3) we have that $\left(a^{\prime}(b r b)+a(b r b)^{\prime}\right) a=0$. By Lemma 3.1, $(b r b)^{\prime}=b^{\prime} r b+b r^{\prime} b+b r b^{\prime}$, so $a(b r b)^{\prime}=a b^{\prime} r b$. Our relation then becomes $\left(a^{\prime} b r b+a b^{\prime} r b\right) a=0$ for all $r \in A$. That is, $\left(a^{\prime} b+a b^{\prime}\right) r b a=0$. Since we have assumed that $b a \neq 0$ and since we are in a prime ring, we are now forced to conclude that $a^{\prime} b+a b^{\prime}=0$. This is the statement of Lemma 3.6, which is now thereby proved.

Corollary. If $a, b \in A$ and if $a b=0$ then $(b a)^{\prime}=b^{\prime} a+b a^{\prime}$.

Proof. By Lemma 3.6, $a^{\prime} b+a b^{\prime}=0$. But $(b a)^{\prime}=(a b+b a)^{\prime}=a^{\prime} b$ $+a b^{\prime}+b^{\prime} a+b a^{\prime}=b^{\prime} a+b a^{\prime}$.

Lemma 3.7. If $a, b \in A$ and if $a b=0$ then for all $c \in A,((b a) c)^{\prime}$ $=(b a)^{\prime} c+(b a) c^{\prime}$.

Proof. By Lemma 3.2, $(c a b+b a c)^{\prime}=c^{\prime} a b+b^{\prime} a c+c a^{\prime} b+b a^{\prime} c+c a b^{\prime}$ $+b a c^{\prime}$. Since $a b=0$, this reduces to

$$
\begin{aligned}
((b a) c)^{\prime} & =b^{\prime} a c+b a^{\prime} c+b a c^{\prime}+c\left(a^{\prime} b+a b^{\prime}\right) \\
& =b^{\prime} a c+b a^{\prime} c+b a c^{\prime} \text { by Lemma 3.6 } \\
& =(b a)^{\prime} c+(b a) c^{\prime} \text { by the corollary to Lemma 3.6. }
\end{aligned}
$$

Let $V=\left\{a \in A \mid(a x)^{\prime}=a^{\prime} x+a x^{\prime}\right.$ for all $\left.x \in A\right\}$. As we have seen by Lemma 3.7, if $a b=0$ then $b a \in V$.

For the sake of convenience of writing we introduce the symbol, for $a, b \in A$, of $a^{b}$ to mean $a^{b}=(a b)^{\prime}=(a b)^{\prime}-a^{\prime} b-a b^{\prime}$.

We note:

$$
a^{b+c}=a^{b}+a^{c}
$$




$$
a^{b}=-b^{a} .
$$

Property (2) is merely a restatement of the fact that ' is a Jordan derivation of $A$. Our aim is to show that $a^{b}=0$ for all $a, b \in A$.

Lemma 3.8. If $t \in V$ and $t \notin Z$ and if $u t=t u$ then $u \in V$.

Proof. By Lemma 3.4

$$
a^{b}(b c-c b)+c^{b}(a b-b a)=0 .
$$

If $t=c \in V$ then $t^{b}=-b^{t}=0$ for all $b \in A$. Thus, in this case, (1) reduces to

$$
a^{b}(t b-b t)=0 \quad \text { for all } a, b \in A .
$$

We linearize (2) with regard to $b$ and obtain

$$
a^{b}(t c-c t)+a^{c}(t b-b t)=0 \quad \text { for all } a, b, c \in A .
$$

If $u t=t u$, putting $b=u$ in (3) we have

$$
a^{u}(l c-c t)=0 \quad \text { for all } a, c \in A .
$$

Thus $a^{u} \in T(t)$. Since $t \in Z$, by Lemma 1.2, $T(t)=(0)$, and so $a^{u}=0$ for all $a \in A$. Thus $u^{a}=0$ for all $a \in A$, from which it follows that $u \in V$.

Lemma 3.9. If $a \in A$ and $a^{2}=0$ then $a \in V$.

Proof. Since $a^{2}=0$, for all $r \in A, a \cdot a r=0$. Thus, by Lemma 3.7, (ar) $a \in V$. If $a=0$ it is clearly in $V$. If we assume that $a \neq 0$, then ara $\neq 0$ for some $r \in A$, by the primeness of $A$. But $a($ ara $)=($ ara $a=0$. Since $\operatorname{ar} a \neq 0,(\operatorname{ara})^{2}=0$ and since $A$ is a prime ring, $a r a \notin Z$. Thus by Lemma 3.8 with $t=a r a \in V$ and $u=a$ we obtain that $a \in V$.

Lemma 3.10. If $c, d \in V$ then $b^{a}(c d-d c)=0$ for all $a, b \in A$.

Proof. As in the proof of Lemma 3.8, since $c, d \in V, b^{a}(c d-d c)$ $+b^{d}(a c-c a)=0$. Since $d \in V, b^{d}=0$ and so $b^{a}(c d-d c)=0$ for all $a, b \in A$ results.

Suppose that $u \in A$ satisfies $u^{2}=0$. By Lemma 3.9, $u \in V$. If, in addition $x^{2}=0, x$ is also in $V$. Thus by Lemma 3.10, $b^{a}(u x-x u)=0$ for all $a, b \in A$. If we multiply this from the right by $u$, using $u^{2}=0$ we obtain

$$
b^{a} u x u=0 \quad \text { for all } a, b \in A .
$$

Now, for any $c, d \in A, c^{d}(c d-d c)=0$, so, for any $r \in A,\left((c d-d c) r c^{d}\right)^{2}$ $=0$. In (1) let $u=(c d-d c) r c^{d}$ and let $x=(a b-b a) s b^{a}$. (1) then becomes 
$(1)^{\prime} \quad b^{a}(c d-d c) r c^{d}(a b-b a) s b^{a}(c d-d c) r c^{d}=0 \quad$ for all $r, s \in A$.

Thus $\left\{b^{a}(c d-d c) r c^{d}(a b-b a)\right\} s\left\{b^{a}(c d-d c) r c^{d}(a b-b a)\right\}=0$, for all $r, s \in A$. Since $A$ is a prime ring we must have that $b^{a}(c d-d c) r c^{d}$ $\cdot(a b-b a)=0$ for all $r \in A$. Thus, since $A$ is a prime ring either $b^{a}(c d$ $-d c)=0$ or $c^{d}(a b-b a)=0$ for all $a, b, c, d \in A$.

In particular, putting $d=b, b^{a}(c b-b c)=0$ or $c^{b}(a b-b a)=0$ for all $a, b, c \in A$.

However, by Lemma $3.4, b^{a}(b c-c b)+c^{b}(a b-b a)=0$. Since, from the above, one or the other of the terms on the left hand side of this equation must always be 0 , we can conclude that each is separately 0 for all choices of $a, b, c$. In consequence we have that $b^{a}(b c-c b)=0$ for all $a, b, c \in A$. Thus, for any $a \in A, b^{a} \in T(b)$. If $b \in Z$ then $T(b)$ $=(0)$ by Lemma 1.2 ; so, in that case $b^{a}=0$ for all $a \in A$. On the other hand, if $b \in Z$, since $b a=a b$ for all $a \in A, b^{a}=0$ by Lemma 3.5. So for all $b$ and $a$ in $A, b^{a}=0$. That is, for all $a, b \in A,(a b)^{\prime}-a^{\prime} b-a b^{\prime}=0$. Thus we have proved

TheOREM 3.1. If $A$ is a prime ring of characteristic different from 2 then any Jordan derivation of $A$ is an ordinary derivation of $A$.

4. Jordan derivations in prime rings of characteristic 2. If one glances through the proofs of the lemmas leading up to Theorem 3.1 one notices that the fact that the characteristic is not 2 is used only in two places, namely in the proof of Lemma 3.1 where we showed that $(a b a)^{\prime}=a^{\prime} b a+a b^{\prime} a+a b a^{\prime}$ and in the proof of Lemma 3.5 when we needed to dispose of the case in which $A$ was commutative.

If we redefine a Jordan derivation of any ring to be:

$$
\begin{aligned}
(a+b)^{\prime} & =a^{\prime}+b^{\prime} ; \\
\left(a^{2}\right)^{\prime} & =a a^{\prime}+a^{\prime} a ; \\
(a b a)^{\prime} & =a^{\prime} b a+a b^{\prime} a+a b a^{\prime}
\end{aligned}
$$

then if the characteristic is not 2 we have not added any restriction in assuming property (3) for it is a consequence of (1) and (2). But now, with this new definition, and the remark of the paragraph above we have

TheORem 4.1. Let $A$ be a prime ring of characteristic 2. Then if $A$ is not a commutative integral domain, any Jordan derivation of $A$ is an ordinary derivation.

YALE UNIVERSITY AND

University of Pennsylvania 